\title{
A CFD study of flow quantities and heat transfer by changing a vertical to diameter ratio and horizontal to diameter ratio in inline tube banks using URANS turbulence models
}

DOI:

10.1016/j.icheatmasstransfer.2017.09.015

\section{Document Version}

Accepted author manuscript

Link to publication record in Manchester Research Explorer

\section{Citation for published version (APA):}

Abed, N., \& Afgan, I. (2017). A CFD study of flow quantities and heat transfer by changing a vertical to diameter ratio and horizontal to diameter ratio in inline tube banks using URANS turbulence models. International Communications in Heat and Mass Transfer, 89, 18-30. https://doi.org/10.1016/j.icheatmasstransfer.2017.09.015

\section{Published in:}

International Communications in Heat and Mass Transfer

\section{Citing this paper}

Please note that where the full-text provided on Manchester Research Explorer is the Author Accepted Manuscript or Proof version this may differ from the final Published version. If citing, it is advised that you check and use the publisher's definitive version.

\section{General rights}

Copyright and moral rights for the publications made accessible in the Research Explorer are retained by the authors and/or other copyright owners and it is a condition of accessing publications that users recognise and abide by the legal requirements associated with these rights.

\section{Takedown policy}

If you believe that this document breaches copyright please refer to the University of Manchester's Takedown Procedures [http://man.ac.uk/04Y6Bo] or contact uml.scholarlycommunications@manchester.ac.uk providing relevant details, so we can investigate your claim.

\section{OPEN ACCESS}




\title{
A CFD study of flow quantities and heat transfer by changing a vertical to diameter ratio and horizontal to diameter ratio in inline tube banks using URANS turbulence models.
}

\author{
Nabeel Abed*, Imran Afgan ${ }^{1}$ \\ * Corresponding author: Nabeel Abed \\ Email: nabilkhalil90@gmail.com \\ Mobile: +9647803021102
}

Mechanical Technical Department, Institute of Anbar, Middle Technical University, Iraq.

1. Imran Afgan

Email: Imran.afgan@manchester.ac.uk

Phone: $+44(0) 1612754334$

School of Mechanical, Aerospace, and Civil engineering, University of Manchester, UK.

\begin{abstract}
This paper reports the effect of changing the aspect ratio on the heat transfer and flow quantities over in-line tube banks. Two types of in-line arrangements were employed; square and non-square configurations. The models that were examined are a standard k- $\varepsilon$ model, SST k- $\omega$ model, v2-f model, EB k- $\varepsilon$ model and EB-RSM model. The closer results to the experimental data and LES were obtained by the EB k- $\varepsilon$ and v2-f models. For the square pitch ratios, the solution has faced a gradual change from a strong asymmetric to asymmetric and then to a perfect symmetry. The strong asymmetric solution was found by the very narrow aspect ratio of 1.2. However, the behaivour of cases of 1.5 and 1.6 became less strong than that predicted in the case of 1.2. In the larger aspect ratio of 1.75, the flow behaviour is seen to be absolutely symmetric for all variables under consideration except Nusselt number. For the very large pitch ratio of 5, the flow has recorded maximum distributions for all parameters on the windward side of the central tube with a perfect symmetric solution around the angle of $180^{\circ}$ while the vortex shedding frequency has recorded minimum value and the Strouhal number; therefore, has given the smallest value. However, for the non-square pitch ratio of constant transverse distance, the solution is still asymmetric for all parameters with merely one stagnation at the angle of $52^{\circ}$ at the case of the $1.5 \times 1.75$ while by increasing the longitudinal distance to 2 and 5 , the solution provided a comprehensive symmetry for all variables with two vortices are fully developed mirrored in shape on the leeward side of the central tube. On the contrary, for the non-square pitch ratio of constant longitudinal distance, the flow of the case of $1.75 \mathrm{X} 1.5$ provided two stagnation locations at around $52^{\circ}$ and $308^{\circ}$ with a very similar solution to the case square ratio of 1.75 for all variables whereas by increasing the transverse distance to 2 and 5, the solution recorded was not perfectly symmetric resulting in two different vortices and one stagnation position located at the leading edge of
\end{abstract}


the cylinder provided by the case of 5X1.5. In terms of vortex shedding effect, the reduction in the Strouhal number at a constant transverse pitch is less steep than those at a constant longitudinal pitch.

Keywords: Heat transfer, Flow quantities, inline tube bundles, URANS turbulence models.

\section{HIGHLIGHTS:}

1- URANS turbulence models in inline tube bundles.

2- The behaviour of flow in square and non-square inline tube bundles.

3- Heat Transfer and vortex shedding in inline configuration.

Corresponding author: Nabeel Abed

Author affiliation: Assistant lecturer

Email: nabilkhali190@gmail.com

Mobile: +9647803021102

Mechanical Technical Department, Institute of Anbar, Middle Technical University, Iraq.

Author

1- Imran Afgan

Author affiliation: Assistant professor

Email: Imran.afgan@manchester.ac.uk

Phone: +44(0)1612754334

School of Mechanical, Aerospace, and Civil engineering, University of Manchester, UK.

\section{Introduction}

The flow through the tube banks has been encountered in a large number of heat exchanger applications in the engineering field in order to exchange the thermal energy (heat) from tube banks as much as possible. However, many numerical and experimental investigations have been made to study the effect of heat transfer, pressure drop, vortex shedding, and vibration over cross flow, parallel flow and oblique flow on both types of flow, laminar and turbulent flows using both configurations; in-line and staggered configurations. The flow can be characterized by transverse and longitudinal pitch-todiameter ratios. By increasing the rows number, the turbulence level and the coefficient of heat transfer have increased significantly and would not be a significant change after a few rows in terms of the level of turbulence, and consequently, the heat transfer coefficient becomes constant. 
Many experimental and numerical investigations have been carried out on both inline and staggered configurations for a wide range of parameters. Experimental data of heat transfer over inline tube banks had been provided by (Aiba et al, 1982). Traub (1990) examined the effect of turbulence intensity on heat transfer and pressure drop on in-line and staggered configurations. Other experimental studies showed the flow regimes in inline and staggered arrangements reported by Ishigai et al. (1973), Zdravkovich (1987) while Lam and Lo (1992) just for an inline configuration. Benhamadouche (2005) and Afgan (2007) have reported numerical investigations using Large Eddy Simulation (LES). Lam, et al. (2008) reported a simulation study on cross flow around square in-line arrangement using finite volume method at Reynolds number of 100 and 200 with a wide range of aspect ratio. In (1992) Lam and Lo carried out an experimental water tunnel investigation to study the frequency of vortex shedding and the wake formation on a four tubes placed in a square arrangement and subjected to cross-flow at a very low Reynolds number. Konstantinidis et al. (2000) studied the water flow around in-line bank tubes in two dimensions, under conditions of steady and pulsating cross flow, using flow visualization and laser Doppler anemometry to provide the detailed characterization of the flow. In (2005) Liang and Papadakis examined the influence of pulsating flow on convective heat transfer and flow field over an array of in-line tubes at Re of 3400, employing the technique of (LES). It was pointed out that with decreasing the aspect ratio; the flow pattern deviated from symmetric to asymmetric. In addition to that, the mean flow has to move in a diagonal direction through the bundle domain. Afgan (2007) and Ladjedel, et al. (2013) carried out the study of turbulent flow over in-line tube bundles by using different models of RANS approach. It was also found that the RANS models gave a good attitude in terms of capturing the transient behaviour of flow. Additionally, it provided good agreement with the experimental measurements. However, only the k- $\varepsilon$ model suppressed the fluctuations of flow.

As presented so far, the gap found in literature survey that need to pay attention for being the change of the transverse pitch (keeping the longitudinal pitch as a constant) and the change of the longitudinal pitch (keeping the transverse pitch as a constant) in a deep study with changing the both pitches in the same investigation at the same Reynolds number which was not covered in the literature. In addition to that, the new turbulence model EB k- $\varepsilon$ would be used in such application which was not used in literature as well. All these issues would be taken into account in more details in the present work. 


\section{Numerical procedure}

\subsection{Flow Periodicity}

The periodic boundary conditions can be described as an assortment of boundary conditions which can be selected in order to discretize very large (infinite) systems by employing a small section from the system. In general, the simulation of tube bundles can be significantly simplified by knowing the fact that the flow repeats itself after passing the entrance by a certain length which in turn leads to allow the flow to be periodic at the certain cyclical boundaries.

The periodic boundary condition as commonly known has a specific condition which is the flow goes out from the one domain is forced to return back to be inflow to another one and thereby the directions would be infinite. Generally, the periodic boundary condition should be specified by either a constant pressure drop or a constant mass flow rate between the inner and outer domains.

\subsection{Computational Mesh Generation}

The present work has been undergone to study the mesh resolution depending on the case of ( $\mathrm{ST} / \mathrm{D}=\mathrm{SL} / \mathrm{D}=1.6)$ and similarly the other subsequent cases have been conducted by considering the similar grid parameters. Four meshes are examined in order to select the more efficient one according to the accuracy of the results and the stability of the solution. These meshes are named; coarse, intermediate, fine and very fine meshes. Sixteen cases are simulated and their cell densities and mass flow rates are summarized in table 1.

Table 1: Number of cells and mass flow rates $(\mathrm{kg} / \mathrm{s})$ of the cases used in the present work.

\begin{tabular}{|c|c|c|c|c|c|c|c|}
\hline \multicolumn{5}{|c|}{ Square pitch ratios } & \multicolumn{3}{|c|}{ Non-square pitch ratios } \\
\hline & \multicolumn{2}{|c|}{$2 \mathrm{D}$} & \multicolumn{2}{|c|}{$3 \mathrm{D}$} & & \multicolumn{2}{|c|}{ 2D } \\
\hline case & $\begin{array}{c}\text { Cell } \\
\text { Density }\end{array}$ & $\begin{array}{c}\text { Mass flow } \\
\text { rate }\end{array}$ & $\begin{array}{c}\text { Cell } \\
\text { Density }\end{array}$ & $\begin{array}{c}\text { Mass } \\
\text { flow rate }\end{array}$ & case & $\begin{array}{c}\text { Cell } \\
\text { Density }\end{array}$ & $\begin{array}{c}\text { Mass flow } \\
\text { rate }\end{array}$ \\
\hline $1.2 \mathrm{X} 1.2$ & 11008 & 0.00011075 & 1100800 & 0.005537 & $1.5 \times 1.75$ & 21504 & 0.000415 \\
\hline $1.5 X 1.5$ & 11840 & 0.0002768 & 1184000 & 0.01384 & $1.5 X 2$ & 27680 & 0.0005537 \\
\hline $1.6 \times 1.6$ & 29952 & 0.000332 & 2995200 & 0.0166 & $1.5 X 5$ & 84032 & 0.002215 \\
\hline $1.75 \times 1.75$ & 66560 & 0.000415 & 6656000 & 0.0207 & $1.75 \times 1.5$ & 21504 & 0.0002768 \\
\hline $5 \times 5$ & 74752 & 0.002215 & 7475200 & 0.11075 & $2 \mathrm{X} 1.5$ & 27680 & 0.0002768 \\
\hline & & & & & $5 X 1.5$ & 84032 & 0.0002768 \\
\hline
\end{tabular}




\subsection{Tube bundle domain}

Many preceding periodic investigations have been done such as (Beale and Spalding, 1999, Benhamadouche et al., 2005 and Afgan 2007) concluded that the domain of 2 X2 has to be enough in terms of capturing both the unsteady flow physics and mean interested mean characteristics. Nevertheless, the domain of 4X4 tubes was investigated numerically by (Benhamadouche et al., 2005 and West 2013) to provide the same flow patterns with another important feature which is there is no difference in the mean characteristics. They also reported that the minimum spanwise direction must be twice the tube diameter $(\mathrm{Lz}=2 \mathrm{D})$ in order to sufficiently cover the flow physics which take place through the inline tube bundles. 


\section{Results and discussion}

\subsection{Mesh independence study}

The most important step in the CFD simulations is to achieve a mesh that creates an opportunity to give more accurate results and faster convergence. The domain corresponding to the square pitch ratio of 1.6 has been selected for the grid independence test. Four grids have been created; and called coarse, intermediate, fine and very fine meshes. The total number of cells in the cross-section for all meshes are 8000, 14480, 29952 and 35712 respectively. Figure 1 illustrates the pressure coefficient distribution around the central tube using four meshes. The more accurate result compared to the very fine grid has been provided by the fine mesh and also the solution is nearly stable as well as the grid provided an independent solution. Therefore, the fine mesh was selected for the present investigation; giving it many pros including save time, accurate results and less computational resources. In terms of wall treatment, the type of (all-y+ wall treatment) has been employed which is available in the STAR CCM+ solver. This type of wall treatment can be described as a hybrid treatment that aims to follow the behaviour of high-wall treatment in the case of a coarse mesh and the behaviour of low-wall treatment in the case of a fine mesh.

\subsection{The turbulence modelling selection}

The square pitch ratio of 1.6 is chosen in order to judge the performance of different RANS models comparing with the measurements of Aiba et al. (1982) at the Reynolds number of 41000 and LES predictions of Afgan (2007) for 2D URANS and 3D URANS calculations. In this section, the two parameters are selected for the comparisons which are the normalized pressure coefficient distribution around the central tube and the normalized velocity profile at the wake of the central column. In addition to that, the flow patterns using velocity streamlines for 2D URANS cases are selected to compare with the LES flow pattern reported by Afgan (2007).

\subsubsection{The normalized pressure coefficient distribution}

Figure 2 shows the normalized pressure coefficient, Cp, profile around the central tube for square pitch ratio of 1.6 for 2D URANS (figure 2a), and 3D URANS (figure 2b), calculations. It is obvious that the experimental data (just available for half cylinder $0^{\circ}-180^{\circ}$ ) and LES results provided high stagnation pressure on the stream-wise direction located around $45^{\circ}$ while the low pressure located at $90^{\circ}$. The LES predicted another low-pressure location at $215^{\circ}$. Fortunately, all the, 2D URANS and 3D URANS calculations seem to provide good agreement with experimental data and LES prediction in some regions like stagnation point and the minimum pressure except the $\mathrm{k}-\varepsilon$ model. However, some of them could not match the experimental data and LES prediction in other regions, especially at the second 
half of cylinder $\left(180^{\circ}-360^{\circ}\right)$. The standard $\mathrm{k}-\varepsilon$ model predicted the stagnation pressure at angle before $45^{\circ}$ in all cases (2D URANS and 3D URANS) and the low pressure predicted is located after $90^{\circ}$ and its value is higher than the experimental data in 2D URANS and also predicted two maximum points in 3D URANS. However, both EB-RSM and SST k- $\omega$ presented good agreement with experimental data and LES results in the first half $\left(0^{\circ}-180^{\circ}\right)$ while gave over-prediction in the 2D URANS around $300^{\circ}$ and their results are approximately symmetric around the $180^{\circ}$. The much closer turbulence model to the experimental data is the $\mathrm{EB} k-\varepsilon$ model which acts to mimic the behaviour of the experimental data in all regions especially in 2D URANS and 3D URANS. The v2-f model shows good agreement in the 2D URANS with the experimental measurements whereas some overpredictions have appeared in 3D URANS in some locations which are around $\left(120^{\circ}-180^{\circ}\right)$ and $\left(300^{\circ}\right.$ $\left.360^{\circ}\right)$.

\subsubsection{The normalized velocity profiles at the wake of the central tube}

The normalized time-averaged velocity profile at the wake of the central column is another parameter considered to compare the URANS predictions in 2D and 3D with the experimental data and LES prediction as observed in figure 3. Figures ( $3 a$ and $3 b$ ) show two peak values in the regions where the mass flow rate is applied (i.e. between the cylinders) and between them, the velocity has decreased due to the presence of the cylinder.

The k- $\varepsilon$ model failed to predict the peaks in the same locations shown by the measurements and LES just in 2D URANS case (figure 3a) while the EB-RSM shows good agreement above the central tube but was far away from the experimental data below the central tube. However, the $\mathrm{k}-\varepsilon$ model gave a better prediction in the 3D case (figure 3b) but still not close to the measurements and LES. Nevertheless, the behaviour of the three rest turbulence models (SST k- $\omega$, v2-f and EB k- $\varepsilon$ ) is approximately the same in all cases and in all regions as well. In all cases, their results are nearly close to the experimental data and LES above $y=0.04 \mathrm{~m}$, whereas below $\mathrm{y}=0.04 \mathrm{~m}$, their behaviour is better and their path is more uniform.

\subsubsection{Flow pattems}

Figure 4 presents the flow patterns predicted by URANS models compared with the LES prediction of Afgan (2007) for the square pitch ratio of 1.6. As can be seen, the periodic, vortex shedding behaviours are predicted by all the 2D URANS models. However, both the k- $\varepsilon$ and EB-RSM models showed symmetrical behaviour by generating two identical bubbles in the recirculation region. The EB-RSM model predicts larger bubbles where they fill the gap between the cylinders, whereas the bubbles predicted by the $\mathrm{k}-\varepsilon$ model are smaller and attached at the top of the cylinder. In such flow (symmetric) one can expect to achieve poor prediction in heat transfer. On the contrary, the v2-f and 
SST k- $\omega$ models predicted approximately the same asymmetric flow behaviour where the large bubble is predicted below the small one behind the central tube whereas behind the first cylinder, the large bubble is predicted above the small one. The most interesting feature is that the EB $k-\varepsilon$ model predicts exactly the same behaviour of LES in all regions in the tube bundle domain where two asymmetrical bubbles are predicted in the recirculation regions. Moreover, the large bubble behind the first tube is predicted below the smaller one as in LES and also it is predicted above the smaller bubble behind the central tube.

To sum up, In spite of the fact that the $k-\varepsilon$ model is valid for most engineering applications with results reasonably accurate, its performance becomes very poor in flow with large pressure gradients, high streamline curvature and strong separation. Moreover, the numerical stiffness is another deficiency in this model where through the viscous sub-layer, the damping function is used which in turn leads to the stability issue. The SST k- $\omega$ renders good results in separation flow and it is highly recommended for simulations where high accuracy boundary layer is required but it has two disadvantages; it is strongly dependent on the wall distance and mesh resolution is required near the wall.

Even though the physical robust of the EB RSM model, it is complex and not easy to converge and it is also not the best model in this study. However, although the v2-f model offers some pros such as more accurate results produced without adding a wall-distance or introducing low-Reynolds number which relates to damping functions in the equations of turbulent kinetic energy, dissipation rate and eddy viscosity, it suffers from the serious drawback of numerical stiffness which in turn leads to cause the weakness of this model with introducing unrealistic results in sometimes. Nevertheless, in the present work, this model has provided good results and one can rank it in the second order after the EB $\mathrm{k}-\varepsilon$. The drawback of the v2-f model is solved in the EB k- $\varepsilon$ model by setting the wall boundary condition of the elliptic variable to zero. The v2-f and EB k- $\varepsilon$ models behave very close to each other and that is not surprising because the EB k- $\varepsilon$ model is the eleventh modified version of the v2-f model and after each modification, the numerical stability effects are carefully appreciated. Therefore, the EB $\mathrm{k}-\varepsilon$ model has achieved considerably more robust result than any other preceding versions. Eventually, the results in the next sections are presented either by the EB k- $\varepsilon$ model or v2-f model or both.

\subsection{Normalized pressure coefficient distribution around the central tube}

The distributions of pressure coefficient, $\mathrm{Cp}=2\left(\mathrm{P}-\mathrm{P}_{\mathrm{ref}}\right) / \mathrm{\rho U}^{2}{ }_{0}$, around the second tube for square in-line tube bundles for all considered gap ratios in 2D and 3D using the v2-f and EB k- $\varepsilon$ models are presented in Figure 5. Figure 5 shows no big difference between 2D and 3D simulation for the same model excluding in the ratio of 1.2 where the difference noticed between around $\left(90^{\circ}-260^{\circ}\right)$ predicted by the EB k- $\varepsilon$ model and also in the ratio of 5 where the difference is very clear between around $45^{\circ}$ - 
$300^{\circ}$ presented by the v2-f model. The discussion below is considered according to the prediction of the EB k- $\varepsilon$ model.

It is observed that the small pitch ratios of 1.2, 1.5 and 1.6 have obtained just a single stagnation point located around $45^{\circ}$ in the direction of flow with two low pressure values located around $90^{\circ}$ and $260^{\circ}$. This is due to the influence of flow deflection. Figure 5a shows the pressure coefficient distribution for pitch ratio of 1.2. Both 2D and 3D simulations for both models provide the stagnation point at the same location which is around $45^{\circ}$. Both models in 2D simulations have presented exactly the same low pressure at angle $90^{\circ}$ while their second low pressure is slightly different from each other at angle of $260^{\circ}$. However, their results are over-estimated for pressure coefficient on the leeward side. The pressure coefficient presented in Figure $5 \mathrm{~b}$ is for pitch ratio of 1.5. When the pitch ratio has relaxed to 1.5, the mass flow rate in the stream-wise direction has been increased due to increasing the cross sectional area according to continuity equation $\left(m=\rho A U_{g}\right)$ and the flow now has an ability to travel in the stream-wise direction easier than in the preceding case. Therefore, that gives the flow an opportunity to record pressure coefficient more uniformly than that recorded by pitch ratio of 1.2.

The better prediction is presented by $3 \mathrm{D} \mathrm{EB} k-\varepsilon$ model because one expects to achieve asymmetric behaviour in such ratio similar to the findings of the LES prediction of Afgan (2007). The same behaviour can be seen in Figure 5c which represents the pitch ratio of 1.6. The pitch ratio now has increased more and the flow behaviour is still nearly asymmetric. The behaviours of v2-f model in 2D and 3D simulations are slowly different from those predicted by EB k- $\varepsilon$ while good agreement is noticed between 2D and 3D simulations predicted by EB k- $\varepsilon$ in all locations.

However, with increasing the pitch ratio for limited value (say <5), the flow is able to show more uniform presentation and nearly symmetric behaviour like the case of 1.75 with two stagnation points at around $50^{\circ}$ and another one around $310^{\circ}$ as presented in Figure $5 \mathrm{~d}$. On the other hand, with continue increasing the pitch ratio to 5 as show in Figure 5e. These two stagnation points became very close to each other where the first one at $0^{\circ}$ while the second one at around $350^{\circ}$ and even in the pressure contour they appear as only one location. This is due to deflection of flow from two locations because the distances between the tubes in transverse and longitudinal directions have significantly increased and; therefore, the flow is no longer restricted to affect the cylinder at the centreline location. Moreover, owing to the flow is specified from two entrances; below and above the cylinders that acts to support each other and meet each other approximately at the centreline location.

The pressure coefficient distributions in the non-square gap ratios are noticed in Figure 6 for 2D simulations using unsteady EB k- $\varepsilon$ model; at a constant transverse distance in Figure $6 \mathrm{a}$ and at a constant longitudinal distance in Figure 6b. For the case of a constant transverse distance, the flow 
behaviour changes moderately from the asymmetric behaviour as shown in the case of $1.5 \mathrm{X} 1.75$ to a symmetric behaviour as noticed in the other cases of $1.5 \mathrm{X} 2$ and $1.5 \mathrm{X} 5$. In addition to that, in the case of 1.5X1.75 the flow deflection acts to influence the stagnation point on the tube just below the cylinder, i.e. only a single stagnation pressure value at around $52^{\circ}$ has appeared. The behaviour of this case is very similar to the case of square gap ratio of 1.5 (figure 5b) with shifting the stagnation pressure from $45^{\circ}$ to $52^{\circ}$ and also increasing the second peak value from 0.34 at angle of $306^{\circ}$ to 0.51 at angle of $302^{\circ}$. By increasing the longitudinal distance to 2 (case of 1.5X2), one can notice the flow deflection now has affected the tube in two locations, i.e. two stagnation values are generated at around $54^{\circ}$ and $305^{\circ}$ and the flow is perfectly symmetric at angle of $180^{\circ}$. By further increasing the longitudinal distance to 5 (case of 1.5X5), the flow behaves exactly the same as the case of $1.5 \mathrm{X} 2$ excluding in the leeward side, where the same stagnation points are noticed at the same locations and the flow is also perfectly symmetric at angle of $180^{\circ}$ while just small difference has been seen in the Cp value between the low pressure locations $\left(90^{\circ}-260^{\circ}\right)$ but the low pressures located at the locations of those generated by the case of $1.5 \mathrm{X} 2$.

On the contrary, in the case of the constant longitudinal distance shown in Figure 6b, the flow physics has clearly changed, especially in the case of further increasing the transverse distance to 5 . Initially, the case of $1.75 \mathrm{X} 1.5$ provided approximately the same behaviour of the square pith ratio of 1.75 (figure 5d) with shifting the stagnation pressure from $50^{\circ}$ to $52^{\circ}$ and also increasing the second peak value from 0.87 at angle of $310^{\circ}$ to 0.95 at angle of $308^{\circ}$. By increasing the transverse distance to 2 , the tube walls became slightly far away from each other and thus the restriction against the flow has been gradually reduced. Consequently, the flow is able to hit the central tube just before the locations recorded by the case of $1.75 \times 1.5$. Thus the first stagnation point located at around $45^{\circ}$ while the second one is shifted to around $315^{\circ}$ and the flow behaviour is still symmetric. By further increasing the transverse distance to 5, the restriction against the flow has been significantly decreased and; therefore, the flow became able to affect the tube around $350^{\circ}-0^{\circ}$ location with nearly symmetrical behaviour is noticed at angle of $180^{\circ}$.

\subsection{Time-averaged velocity profiles}

The time-averaged velocity distributions divided by the mean velocity at the wake of the central column of the tubes (see figure $3 \mathrm{c}$ at $\mathrm{x}=\mathrm{c}$ ) for square inline configurations are presented in Figure 7. The gap velocity is constant for all cases (square and non-square) because it depends on the Reynolds number and tube diameter. Both of them are considered to be constant in this study while the freestream velocity $\left(\mathrm{U}_{0}\right)$ is different in each case, as given in table 2, depending on longitudinal distance $\left(\mathrm{S}_{\mathrm{T}}\right)$. Therefore, it was selected to be denominator of the relation shown in Figure 7. As a result, one can expect to notice a gradual decrease in the velocity gradients as the pitch ratio is relaxed. The 
velocity distributions provided $\mathrm{W}$-profile approximately in all cases which suggest reverse flow in the recirculation regions.

The results are represented for 2D and 3D simulations by two turbulence models; v2-f and EB k- $\varepsilon$. For all gap ratios except the case of $5 \times 5$, the results showed good agreement between turbulence models in all regions except in the recirculation regions. This might be due to the actions generated by the vortex shedding behind a cylinder which might be slightly different in each turbulence model. However, in the case of $5 \times 5$, the EB $k-\varepsilon$ model provided results better than those predicted by the v2-f model, where the velocity in $2 \mathrm{D}$ and $3 \mathrm{D}$ are predicted uniformly and the results of $2 \mathrm{D}$ are very close to those of 3D and also gave approximately a symmetric behaviour at $y=0.125 \mathrm{~m}$. In the discussion below, the $3 \mathrm{D}$ cases predicted by the EB $\mathrm{k}-\varepsilon$ model is considered.

It is noticed that the pitch ratio plays a very important role in terms of the deflection of the maximum stream-wise velocity, when the pitch ratio is increased; the velocity deflection from the centreline is decreased. That was also concluded by Afgan (2007). The deviation for the pitch ratio of 1.2 was very strong and did not take a place in the centreline of the flow (see figure (7a)). This is could be due to the narrow space between tubes that does not allow the flow to move easily and; therefore, when the flow hit the tube, the flow is forced to be deviated from the centreline leading to creating maximum deviated behaviour. However, when the pitch ratio has increased to 1.5, the mass flow rate has been increased due to an increase of area while the velocity gradient became less steep than those of the previous case. Consequently, one can easily notice two things in Figure (7b); the deviation of the velocity stream is significantly reduced and the flow tries to be symmetric. The second observation is that the thickness of the profile between tubes became bigger due to increasing the pitch ratio which require bigger mass flow rate and; therefore, the maximum ratio of $\left(\mathrm{U} / \mathrm{U}_{0}\right)$ decreased from 3.8 to 3.2. In spite of the fact that the mass flow rate has increased in case of 1.6, no huge difference is shown in Figure $6 \mathrm{c}$ with surely reducing the velocity gradient further more. However, by further increasing the pitch ratio to 1.75 shown in Figure (7d), more uniform behaviour is presented and the flow acts to be symmetric at the midway of the longitudinal distance. Another feature is that, the deviation of the mean velocity became close to zero and; as a result, the averaged velocity has collapsed at the centreline, all the previous cases are in good agreement with findings of Aiba et al. (1982) and Afgan (2007). The same behaviour with increasing the pitch ratio to 5 is presented in Figure (6e).

The time-averaged velocity profiles for $2 \mathrm{D}$ non-square configurations at a constant longitudinal distance using the EB k- $\varepsilon$ model at the wake of the central column of the tubes are shown in Figure 8. The flow behaviour has clearly changed, especially in the case of further increasing the transverse distance to 5 . Initially, the case of $1.75 \times 1.5$ provided approximately the same behaviour of the square pith ratio of 1.5 (figure $7 \mathrm{~b}$ ) with slightly reducing the maximum ratio of $\left(\mathrm{U} / \mathrm{U}_{\mathrm{o}}\right.$ ) while the profile 
thickness still the same because the longitudinal distance is taken as a constant. By increasing the transverse distance to 2, the tube walls became slightly far away from each other and thus the flow is relaxed which in turn led to decrease the maximum ratio of $\left(\mathrm{U} / \mathrm{U}_{0}\right)$. By further increasing the transverse distance to 5 , the flow is considerably relaxed under the same mass flow rate. That led to giving nearly constant linear behaviour and even the effect of the tube has disappeared. That might be the vortex shedding became far away from the central distance between the tubes and; therefore, its effect became very weak.

On the other hand, the time-averaged velocity profiles for 2D non-square configurations at a transverse distance using the EB k- $\varepsilon$ model are presented in Figure 9. The case of 1.5X1.75 (figure 9a) tends to provide small deviation for the maximum velocity gradient. It is interesting to note that both other cases of $1.5 \mathrm{X} 2$ and 1.5X5 provided perfect symmetric behaviour at the midway of longitudinal distance. Additionally, the velocity deviations for both cases have collapsed in the centreline. Another observation one can consider is that the maximum ratio of $\left(\mathrm{U} / \mathrm{U}_{0}\right)$ has decreased moderately from 2.5 at $1.5 \mathrm{X} 1.75$ to 2.3 at $1.5 \mathrm{X} 2$ and finally to 1.45 at $1.5 \mathrm{X} 5$.

Table 2: Free-stream velocities for all cases in the present work.

\begin{tabular}{|l|l|l|l|l|l|}
\hline \multicolumn{3}{|l|}{ Square pitch ratios } & \multicolumn{3}{l|}{ Non-square pitch ratios } \\
\hline No & Case & $\mathrm{U}_{\mathrm{o}}(\mathrm{m} / \mathrm{s})$ & No & Case & $\mathrm{U}_{\mathrm{o}}(\mathrm{m} / \mathrm{s})$ \\
\hline 1 & $1.2 \mathrm{X} 1.2$ & 1.5893 & 1 & $1.5 \mathrm{X} 1.75$ & 4.0860 \\
\hline 2 & $1.5 \mathrm{X} 1.5$ & 3.1786 & 2 & $1.5 \mathrm{X} 2$ & 4.7680 \\
\hline 3 & $1.6 \mathrm{X} 1.6$ & 3.5700 & 3 & $1.5 \mathrm{X} 5$ & 7.6288 \\
\hline 4 & $1.75 \mathrm{X} 1.75$ & 4.0860 & 4 & $1.75 \mathrm{X} 1.5$ & 3.1786 \\
\hline 5 & $5 \mathrm{X} 5$ & 7.6288 & 5 & $2 \mathrm{X} 1.5$ & 3.1786 \\
\hline & & & 6 & $5 X 1.5$ & 3.1786 \\
\hline
\end{tabular}

\subsection{Flow pattems}

The flow pattern of fluid can easily characterize the pressure drop and heat transfer in tube bundles. However, the flow patterns can be strongly affected by boundary conditions, a number of tubes used in the cluster and the pitch ratios and become fairly complex when the transverse and longitudinal distances between the tube centres are very small. This is due to the mutual interference of the flow which leads to cause a presence the interesting and unexpected phenomena. The tube bundle is different from a single tube in terms of the flow pattern because in tube bundles, the tube is located in the another's wake and; therefore, the flow physics are completely changed and the behaviour of a tube downstream become strongly depending on the flow pattern in the wake of a tube upstream. 
For the square pitch ratios, the nature of the flow through in-line tube banks can be described depending on the pitch ratios by two features; unsuitability and asymmetry for small pitch ratios while the stability and symmetry tend to take place as the pitch ratio becomes bigger. The time-averaged velocity streamlines for $2 \mathrm{D}$ square pitch ratios achieved using the $\mathrm{EB} \mathrm{k}-\varepsilon$ model are presented in figure 10. The gap ratio of 1.2 can be noticed from figure 10a where an asymmetric behaviour and complete unidirectional flow are presented. In addition to that, for the central tube, two vortex shedding are taking place at the top leeward side and the shear layer has separated from the top of the central tube with generating two recirculation zones. Another feature one can be taken into account which is the fluid acts to flow diagonally among the tubes instead of following in the stream-wise direction. This could be occurred in order to avoid the effects introduced from the abrupt expansions and reductions, which in turn lead to lose less energy than in the stream-wise direction.

The flow pattern predicted in the pitch ratio of 1.5 shown in figure 10b, the flow physics have changed and the shear layer now separates from the bottom leeward side with creating two different recirculation zones just behind the tubes in alternating asymmetrical behaviour. As the pitch ratio further increases to 1.6 presented in figure 10c, the same behaviour is achieved with very important feature one can focus on which is the bubble sizes have gradually changed which tend to be getting ready for the similarity in the further relaxation in the pitch ratio. In the pitch ratio of 1.75 noticed in figure 10d, the flow now completely changed to be absolutely symmetrical behaviour with also two recirculation zones generated behind every tube. By further increasing the pitch ratio to 5 illustrated in Figure 10e, the flow again took asymmetric bubbles; however, these bubbles are not able to fill the whole regions behind the tubes due to a big distance.

Figure 11 shows the time-averaged velocity streamlines for $2 \mathrm{D}$ non-square pitch ratios at a constant longitudinal distance using the EB k- $\varepsilon$ model. If one compares the square pitch ratio of 1.5 shown in figure 13b with the case of $1.75 \times 1.5$ observed in Figure 11a, the flow is still denominated by the asymmetrical behaviour with something is interesting can be noted which is the bubble sizes became bigger and also filled the recirculation regions. However, when the transverse distance relaxed to 2 as observed in figure 11b, both bubble sizes have clearly changed where the small bubble in the case of 1.75X1.5 has obtained a further reduction in the case of 2 X1.5 whereas the large bubble in the case of 1.75X1.5 has achieved a further increase in the case of $2 \mathrm{X} 1.5$ in spite of the fact that the same mass flow rate was specified for all of them. Nevertheless, by increasing the transvers distance to 5 as shown in 11c, the flow behaviour is still alternating asymmetrical but now the larger bubble in the previous case became the smaller bubble in this case and the smaller bubble in the preceding case became the larger bubble in this case. 
On the other side, the time-averaged velocity streamlines for $2 \mathrm{D}$ non-square pitch ratios at a constant transverse distance predicted by the EB $\mathrm{k}-\varepsilon$ model are observed in figure 12. By looking to the square ratio of 1.5 and compare it with the case of $1.5 \mathrm{X} 1.75$, the flow is still controlled by the asymmetrical behaviour with something important can be noted which is the bubble sizes became completely different where the larger bubble behind the first cylinder became the smaller at the case of $1.5 \mathrm{X} 1.75$ while the smaller bubble became the larger in the case of $1.5 \times 1.75$ and vice versa behind the second cylinder. By increasing the longitudinal distance to 2 and 5, the flow provided absolutely perfect symmetric behaviour with a more uniform profile.

\subsection{The Nusselt number distribution around the second tube}

In general, the calculations of the Nusselt number of the in-line tube bundles are strongly dependent on three variables. These are number of rows in the domain, Reynolds number and the surface temperature (Ts). However, all of these variables are set to be constant in the present work. Therefore, approximately all the maximum values shown in figure 13, are close to each other while the locations of them are different due to the flow deflection through tube banks.

The distributions of time-averaged Nusselt number $(\mathrm{Nu})$ around the central cylinder for the 3D square pitch ratios using the EB k- $\varepsilon$ model are represented in figure 13a. For the square pitch ratio of 1.2, the share layer separated from the upstream cylinder has reached the second tube at around $30^{\circ}$ resulting in the maximum $\mathrm{Nu}$ in this location with nearly an asymmetric profile. In this aspect ratio, the flow has deflected upward completely. The separated shear layer is responsible for providing the mutual interference which aims to cause the flow deflection through the narrow gap ratio. However, for the ratio of 1.5 , because the space between the tubes became bigger and the flow is able to move with less restriction than in the previous case and; consequently, the maximum value now shifted to $51^{\circ}$. That gives an idea that the flow tries to be symmetric but needs further relaxation in the pitch ratio. The similar behaviour was achieved by increasing the pitch ratio to 1.6 and both of them have faced the second maximum value at around $300^{\circ}$. Nevertheless, for the gap ratio of 1.75 , both of the maximum values now increased while they are staying in the same positions provided by the gap ratios of 1.5 and 1.6. However, in terms of symmetry, this gap ratio seems to be better. By further increasing the aspect ratio to 5, the space between the tubes became very enough for flow to be deflected resulting in a perfect symmetry at the angle of 180 . That means the heat transfer becomes larger on the windward side and reduces to the minimum values on the leeward side.

The $\mathrm{Nu}$ of the non-square aspect ratio in $2 \mathrm{D}$ at a constant transverse distance using the EB k- $\varepsilon$ model is presented in figure 13b. For the initial case of $1.75 \mathrm{X} 1.5$, the profile is not perfect symmetry while by 
further increasing the longitudinal distance to 2 and 5 , the $\mathrm{Nu}$ profiles become absolutely symmetric and the maximum values have recorded at the same locations which around $50^{\circ}$ and $305^{\circ}$.

On the other side, the $\mathrm{Nu}$ of the non-square aspect ratio in 2D at a constant longitudinal distance using also the EB k- $\varepsilon$ model is illustrated in figure 13c. The flow physics has now been changed and the Nu distributions around the central tube have changed as well. As can be seen from figures 13c, with increasing the transverse distance from 1.75 to 2 , both peaks have slightly shifted from $48^{\circ}$ and $308^{\circ}$ in the case $1.75 \times 1.5$ to $43^{\circ}$ and $310^{\circ}$ in the case $2 \mathrm{X} 1.5$. In addition to that, the Nu profile tries to be symmetry but it is not. By further increasing the transverse distance to 5, the flow has now been able to provide a perfect symmetry at around $180^{\circ}$ and the Nu distribution has concentrated just on the windward side of the central tube $\left(360^{\circ}-0^{\circ}\right)$.

\subsection{Vortex shedding}

Vortex shedding is a very important aspect of the tube bundle system.It can be described as an unsteady process in which the turbulent eddies can be transported from the trailing edge of the tube in the pattern of vortices. Figure 14 presents the variation of stream-wise velocity with time at a point just behind the central tube in the case of square pitch ratio of 1.6. As can be seen from the same figure, the cyclic variation with time is owing to the cyclic vortices generated behind the tube. For this vortex shedding, it is noticed that the period of time is nearly $0.0225 \mathrm{sec}$ and the corresponding frequency is 44.4 Hz. Therefore, the non-dimensional Strouhal number can be computed by multiplying the frequency by the tube diameter and the free-stream velocity. Similarly, the Strouhal numbers of all cases are computed by the same procedure and their values are given in table 3 .

In general, Strouhal number depends on several parameters includes tube spacings, Reynolds number, and turbulence intensity. However, the Reynolds number is considered to be constant here. Therefore, the Strouhal number in the present work only depends on tube spacings and turbulence intensity. The Strouhal numbers of square pitch ratios in 3D are shown in figure 20 compared with the measurements of Fitz-Hugh (1937). The results showed a close relation to the experimental data in all cases except in very narrow gap ratio of 1.2 where the numerical prediction provided over-prediction in this case. As can be seen, the very small ratio of 1.2 has recorded a very large Strouhal number of 0.461 . This is due to the tube space through the bank, which is very narrow and thereby the vortex shedding needs very small time to complete a period of time. Therefore, the frequency recorded very high corresponding to very small time and; consequently, the very large Strouhal number occurred as physically expected. The smaller the square pitch ratios, the larger the Strouhal number would occur. 
However, when the square pitch ratio has been relaxed to 1.5, 1.6, 1.75 and 5, the space between tubes has gradually increased and turbulence accordingly decreased. Therefore, the frequency of vortex shedding became smaller and Strouhal number accordingly decreased.

For non-square pitch ratios in both cases of constant transverse and longitudinal pitches, the Strouhal numbers decreased with increasing the pitch ratio. There is a very important feature one can appreciate which is the reduction in Strouhal number at a constant transverse pitch is less steep than those at a constant longitudinal pitch. This means that the effect of the cylinder wake is larger than the effect of the longitudinal distance between cylinders.

Table 3: Strouhal numbers of all cases using the EB k- $\varepsilon$ model.

\begin{tabular}{|l|l|l|l|l|l|}
\hline \multicolumn{3}{|c|}{ Square pitch ratios (3D) } & \multicolumn{3}{l|}{ Non-square pitch ratios (2D) } \\
\hline No. & Case & St. number & No. & Case & St. number \\
\hline 1 & $1.2 X 1.2$ & 0.461 & 1 & $1.5 X 1.75$ & 0.332 \\
\hline 2 & $1.5 X 1.5$ & 0.342 & 2 & $1.5 X 2.0$ & 0.315 \\
\hline 3 & $1.6 X 1.6$ & 0.311 & 3 & $1.5 X 5.0$ & 0.195 \\
\hline 4 & $1.75 X 1.75$ & 0.298 & 4 & $1.75 X 1.5$ & 0.325 \\
\hline 5 & $5.0 X 5.0$ & 0.230 & 5 & $2.0 X 1.5$ & 0.251 \\
\hline & & & 6 & $5.0 X 1.5$ & 0.181 \\
\hline
\end{tabular}




\section{Conclusion}

Computational investigations were performed for square and non-square in-line tube bundles to study the effect of changing the aspect ratios on the pressure coefficient distribution, velocity profile, turbulence intensity, flow patterns, Nusselt number distribution, and vortex shedding. The square pitch ratios are $1.2 \mathrm{X} 1.2,1.5 \mathrm{X} 1.5,1.6 \mathrm{X} 1.6,1.75 \mathrm{X} 1.75$, and $5 \mathrm{X} 5$. All of them have been studied in $2 \mathrm{D}$ and $3 \mathrm{D}$. While the non-square gap ratios are $1.5 \mathrm{X} 1.75,1.5 \mathrm{X} 2,1.5 \mathrm{X} 5,1.75 \mathrm{X} 1.5,2 \mathrm{X} 1.5$, and $5 \mathrm{X} 1.5$. All of them were investigated in 2D. Five turbulence models were tested, namely (EB RSM, SST k- $\omega$, standard $\mathrm{k}-\varepsilon$, v2-f and EB $\mathrm{k}-\varepsilon$ ) and after comparing the results of the square ratio of 1.6 with the experimental data of Aiba et al. (1982) and the LES prediction of Afgan (2007), the best turbulence

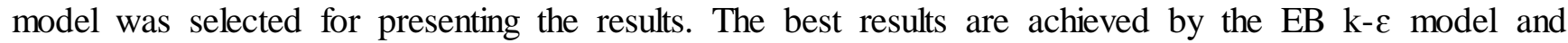
the v2-f model also provides good results. Therefore, the results are presented by either the EB k- $\varepsilon$ model or both of them.

\subsection{The square pitch ratios}

For the very small aspect ratio of 1.2 , the solution achieved is a very strong asymmetric for the parameters under consideration such as pressure coefficient, Nusselt number distribution. In addition to that, the deviation of the mean flow has recorded high level from the cylinder centreline as presented in the velocity profile and turbulence intensity. However, for the medium cases of 1.5 and 1.6, the solution is still providing asymmetric behaviour in all parameters but less strong than that predicted in the case of 1.2. That might be because the blockage influences coming from the downstream tubes which act to highly destroy the vortex shedding in these medium cases and; as a result, two vortices different in size are generated as presented in the time-averaged velocity field. In addition to that, for all previous cases, the locations of the separation shear layer are completely different from each other which in turn led to provide an alternating asymmetrical behaviour as presented in the flow patterns. In the larger aspect ratio of 1.75, because the space between tubes became bigger, this in turn led to decrease the blockage influences. Therefore, the flow behaviour is seen to be absolutely symmetric for all variables under consideration except Nusselt number. Additionally, the locations of the separation shear layer are completely the same with each other resulting in a perfect symmetric solution with two equivalent vortices but in a mirrored shape as presented in the flow patterns and the lengths of the both bubble regions are exactly the same as predicted on the leeward part of the central tube. For the very large pitch ratio of 5, the flow has recorded maximum distributions for all parameters on the windward side of the central tube with a perfect symmetric solution around the angle of $180^{\circ}$. The Strouhal number has recorded maximum values in the very narrow gap ratio of 1.2 and gradually reduced as the pitch ratios have been relaxed.

\subsection{The non-square pitch ratio}




\section{A. At the constant transverse distance:}

The flow at a moderate longitudinal distance of 1.75 is still asymmetric for all variables with only one stagnation point at the angle of $52^{\circ}$ and the velocity profile suffers from the deviation in the centreline. However, by increasing the longitudinal distance to 2 and 5, the blockage effect in this direction became very small. Therefore, the flow was able to generate a perfect symmetry for all parameters (pressure coefficient, velocity profile, turbulence intensity, turbulent kinetic energy, flow patterns and Nusselt number) and fully two vortices mirrored in shape are developed and exactly the same bubble lengths are created on the leeward side of the tubes. Moreover, the flow generates the same two stagnation points at $54^{\circ}$ and $305^{\circ}$. It can be understood that the transverse distance is responsible for giving symmetrical behaviour by increasing the longitudinal distance to 2 and upwards.

\section{B. At the constant longitudinal distance:}

The flow at a moderate transverse distance of 1.75 is similar to the square ratio of 1.75 for all variables with two stagnation points at the angles of $52^{\circ}$ and 308. However, by increasing the transverse distance to 2 and 5, although the blockage effect in this direction became very small, the flow was not able to generate a perfect symmetrical behaviour for all parameters and; therefore, two different vortices are developed on the leeward side of the tubes. Moreover, the stagnation points have shifted further from above and below the tube to the zero-degree location resulting in only one stagnation point. It can be detected that the longitudinal distance is responsible for bringing the two stagnation points very close to each other resulting in only one stagnation point. In terms of Strouhal number, the reduction in Strouhal number at a constant transverse pitch is less steep than those at a constant longitudinal pitch.

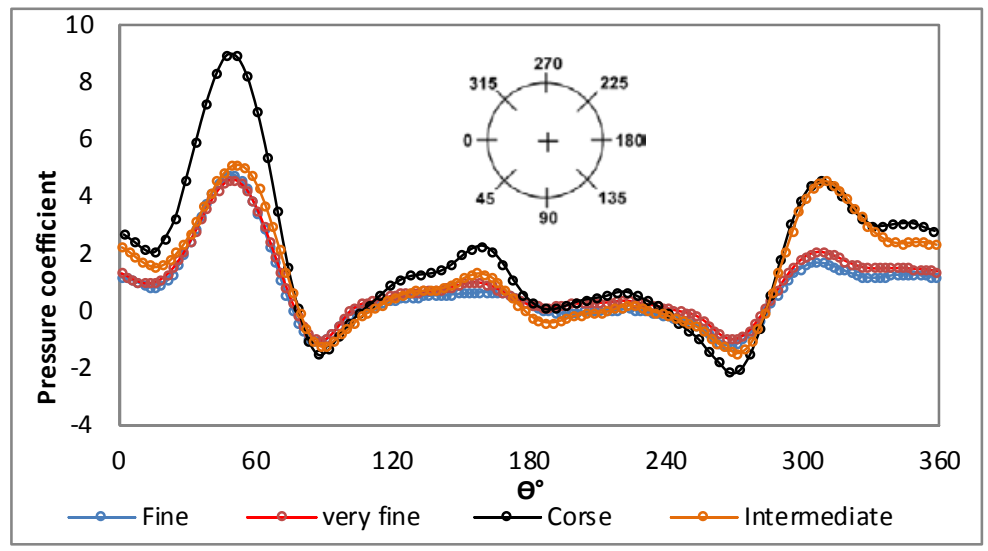

Figure 1: Pressure coefficient distribution around the central tube of the case of $1.6 x 1.6$ for all grids. 


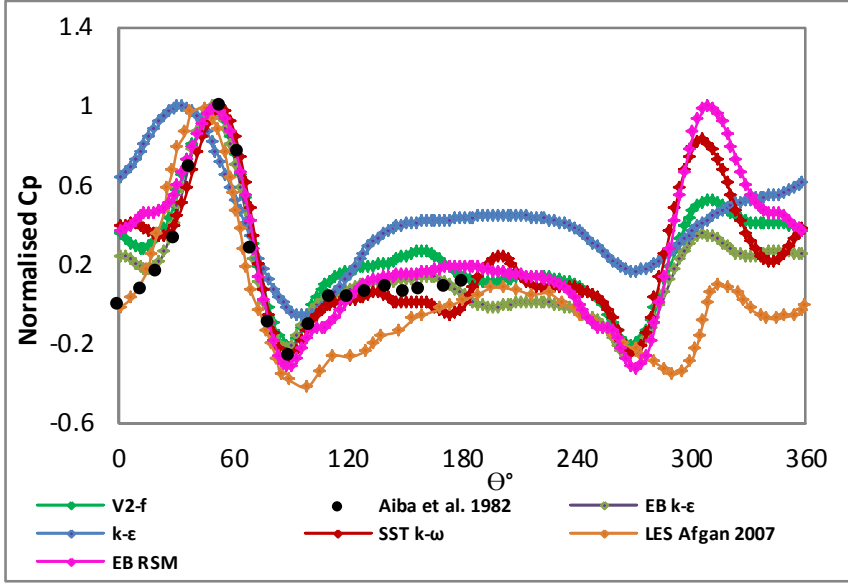

(a) 2D URANS

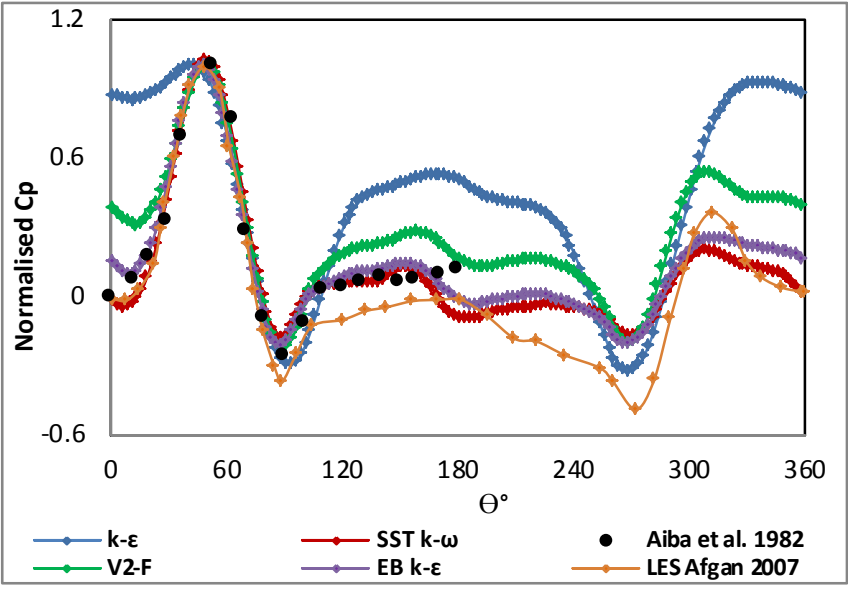

(b) 3D URANS

Figure 2: The normalized pressure coefficient around the central tube compared with experimental data of Aiba et al. (1982) and LES of Afgan (2007).

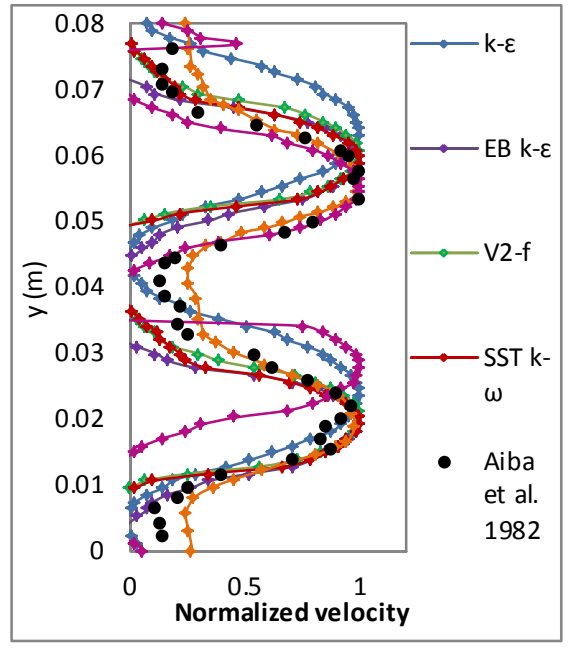

(a)

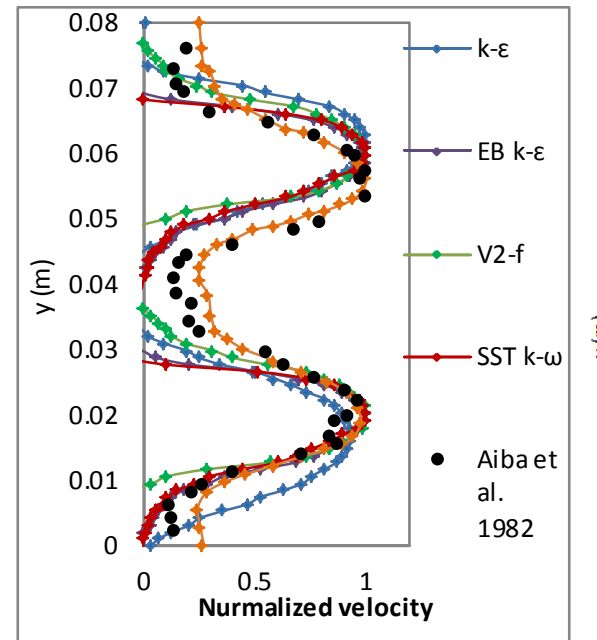

(b)

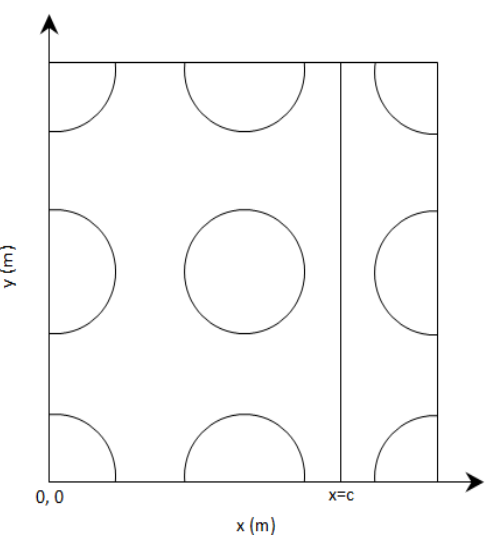

(c)

Figure 3: A comparison between RANS models and experimental data of Aiba et al. (1982) and LES of Afgan (2007) using normalized mean velocity in the wake of central column; (a) 2D URANS, (b) 3D URANS, and (d) a schematic diagram shows the certain location. 


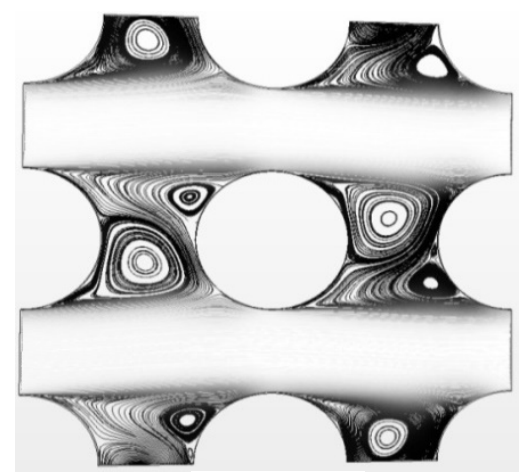

EB $k-\varepsilon$

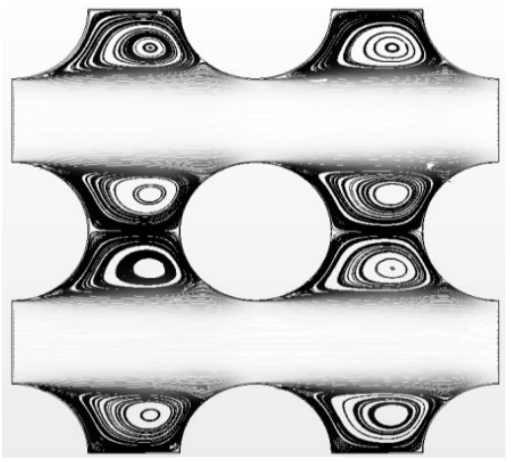

EB RSM

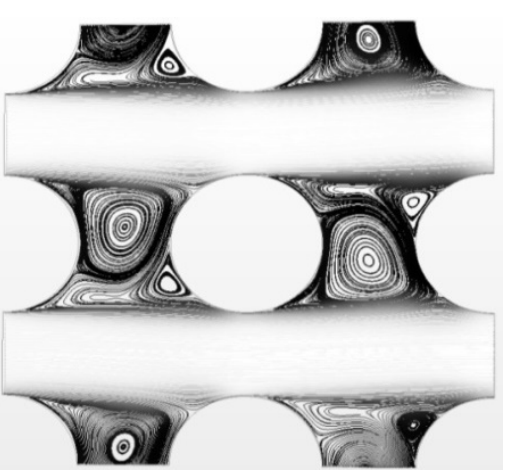

V2-f

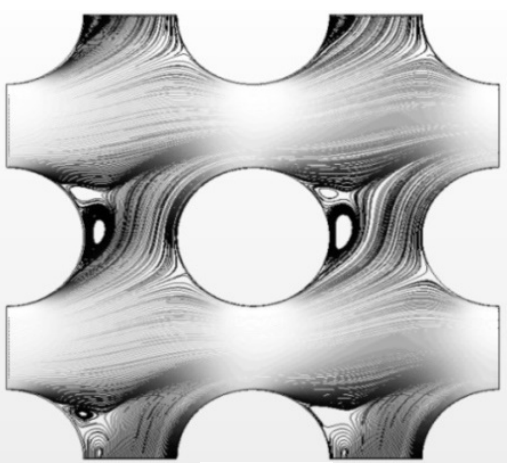

$\mathrm{k}-\varepsilon$

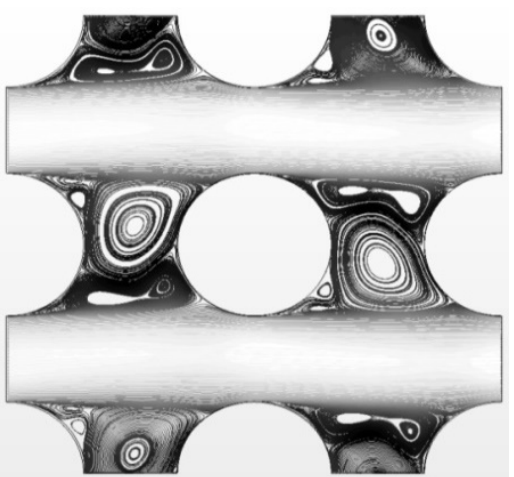

SST k- $\omega$

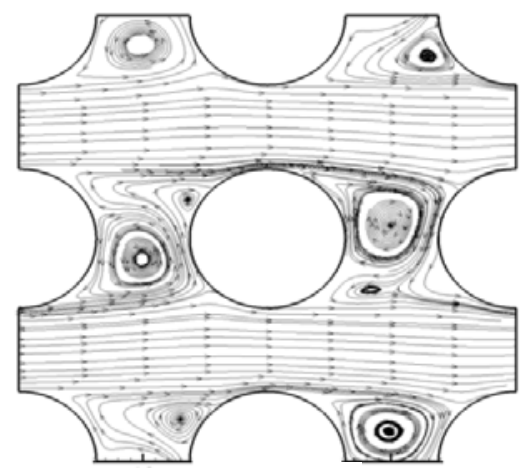

LES

Figure 4: A comparison between 2D URANS and LES of Afgan (2007) using mean velocity streamlines. 


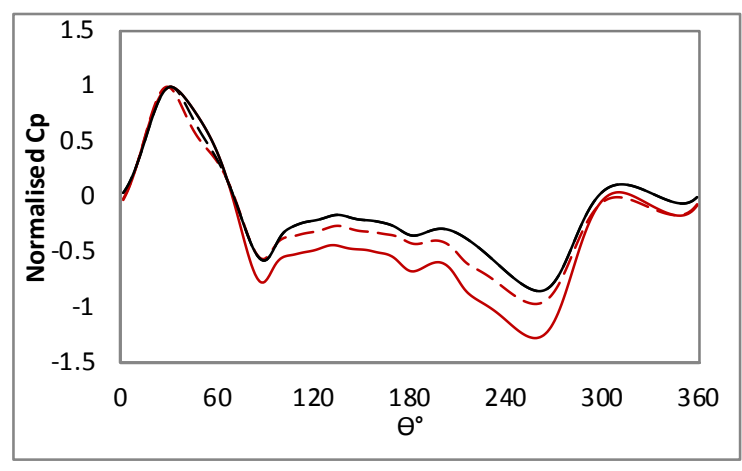

(a) $1.2 \times 1.2$

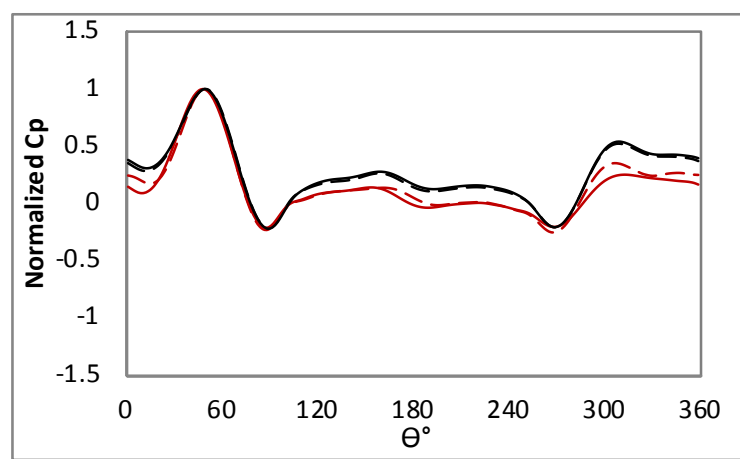

(c) $1.6 \times 1.6$

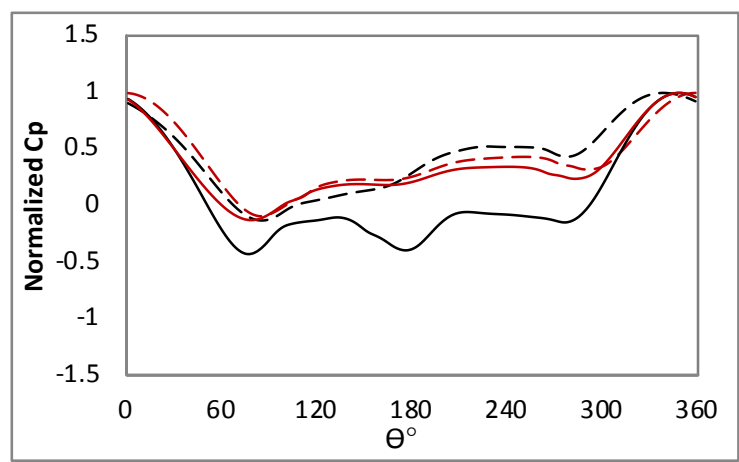

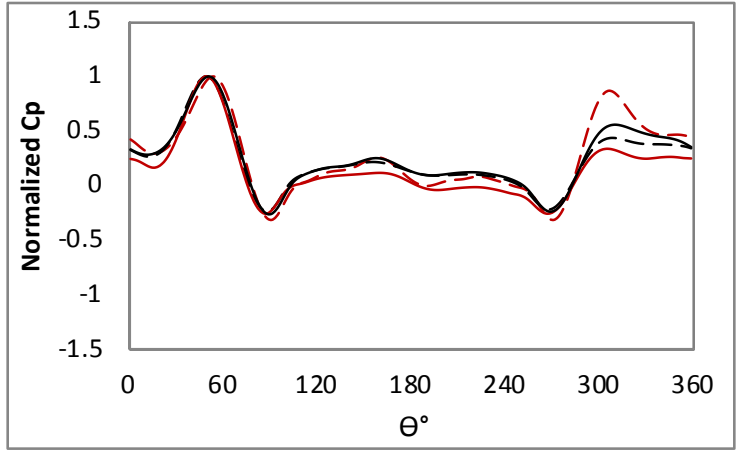

(b) $1.5 \times 1.5$

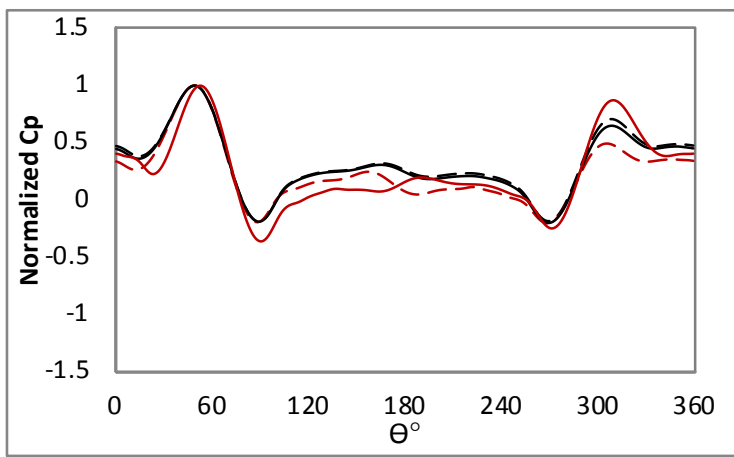

(d) $1.75 \times 1.75$

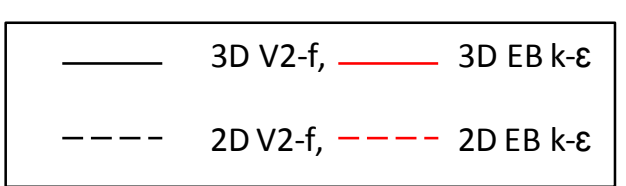

(e) $5 \times 5$

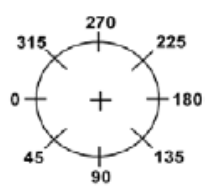

Figure 5: The normalized pressure coefficient around the central tube in square in-line tube bundle using URANS.

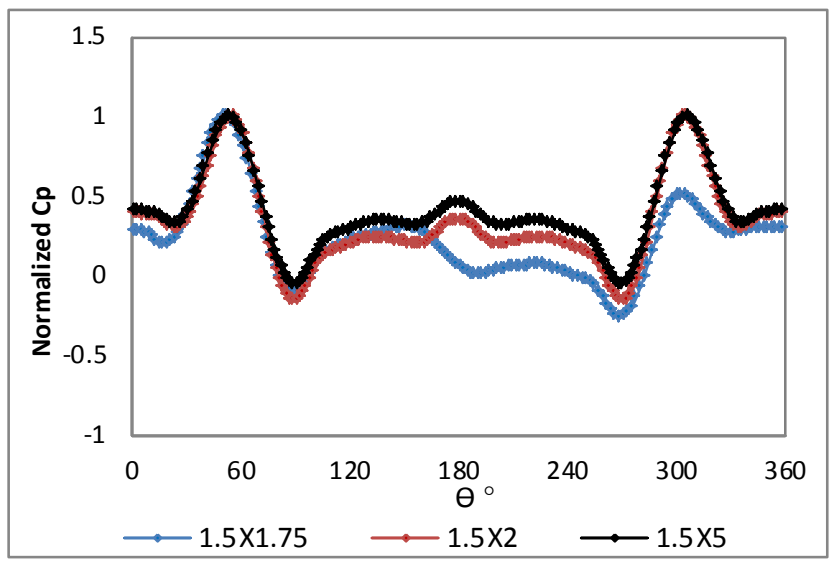

(a) At a constant transverse

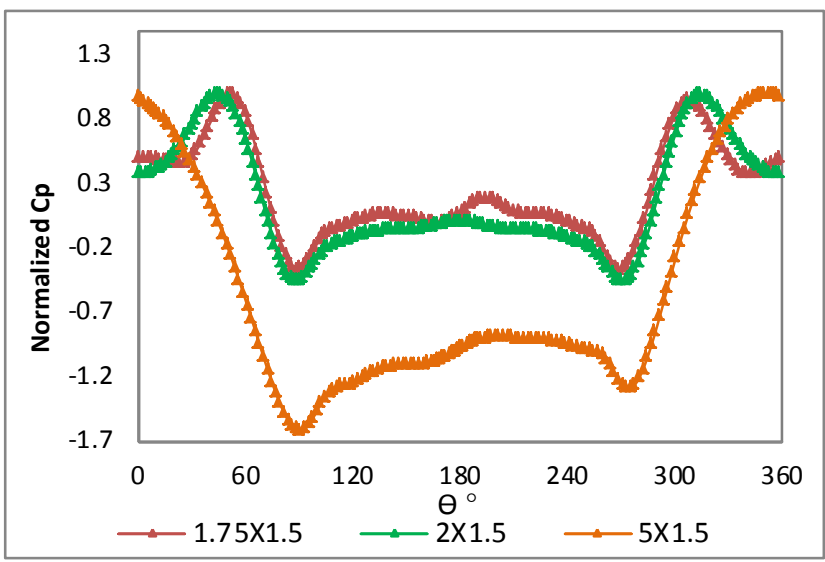

(b) At a constant longitudinal distance

Figure 6: The normalized pressure coefficient (Cp) around the central tube in non-square in-line tube bundle using 2D unsteady EB k- $\varepsilon$ model. 


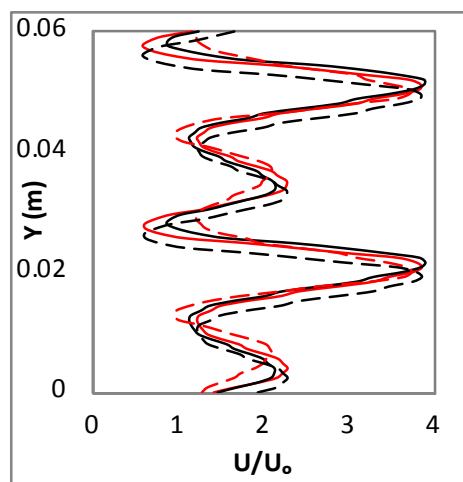

(a) $1.2 \times 1.2$

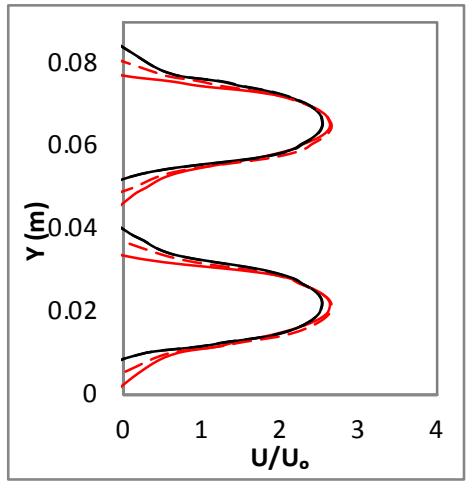

(d) $1.75 \times 1.75$

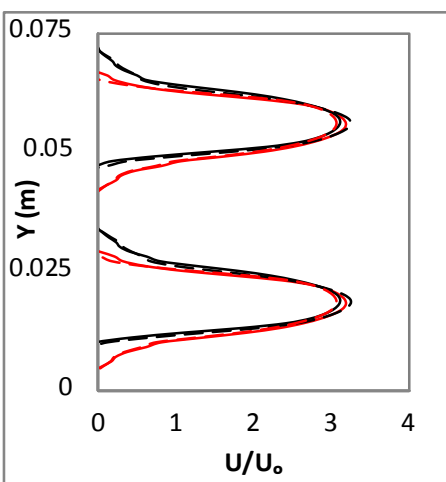

(b) $1.5 \times 1.5$

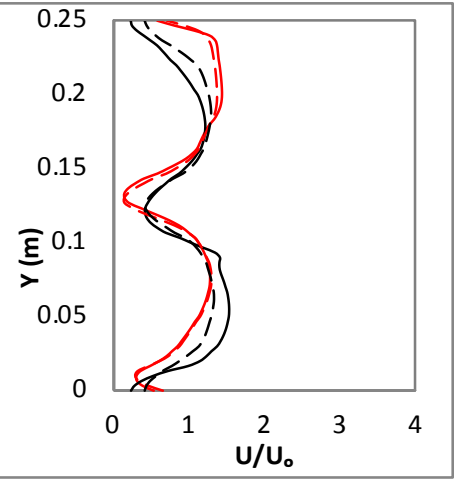

(e) $5 \times 5$

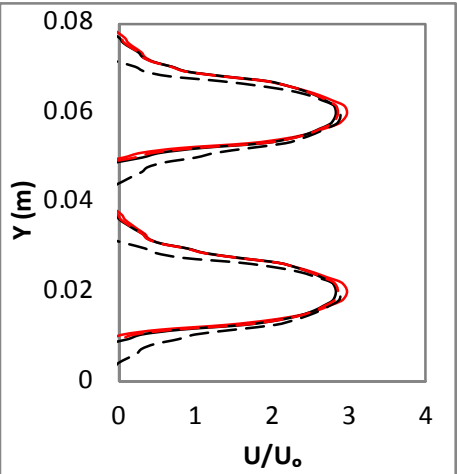

(c) $1.6 \times 1.6$

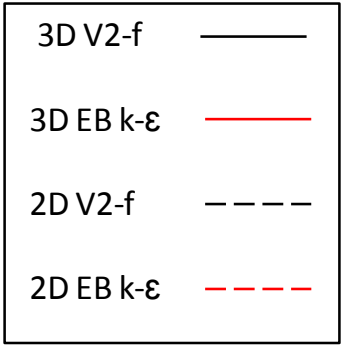

Figure 7: Time-averaged velocity profile at the wake of the central column for unsteady, 2D and 3D square configurations.

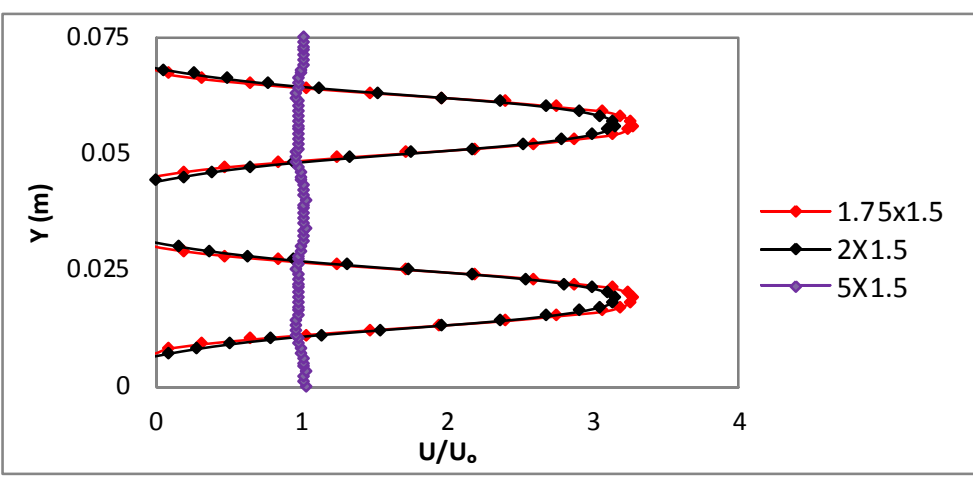

Figure 8: Time-averaged velocity profile at the wake of the central tube for $2 \mathrm{D}$ non-square configurations at constant longitudinal distance using unsteady EB k- $\varepsilon$ model.

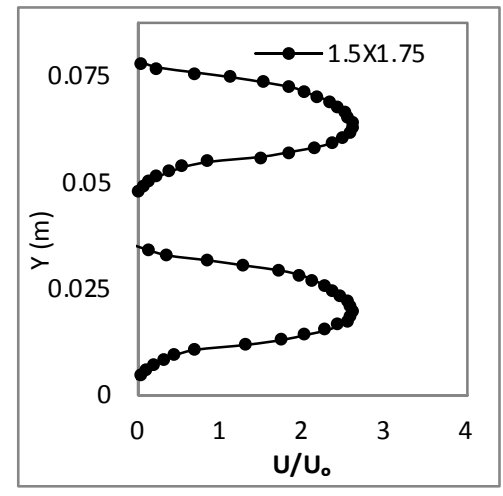

(a) $1.5 \times 1.75$

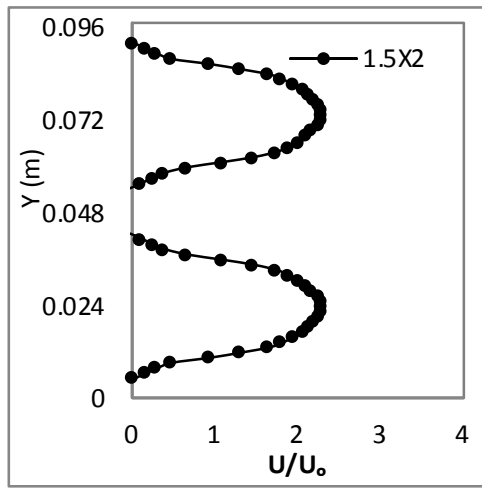

(b) $1.5 \times 2$

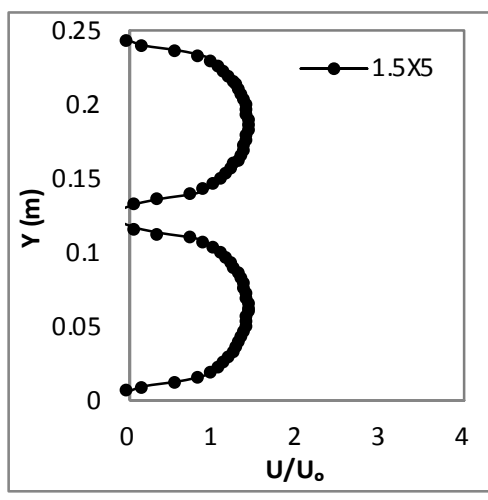

(c) $1.5 \times 5$

Figure 9: Time-averaged velocity profile at the wake of the central tube for $2 \mathrm{D}$ non-square configurations at a constant transverse distance using unsteady EB k- $\varepsilon$ model. 


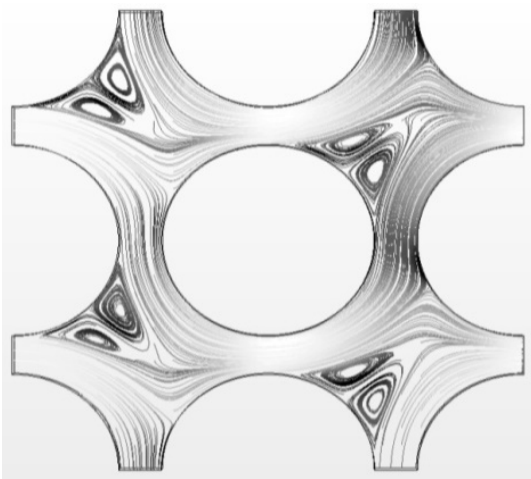

(a) $1.2 \times 1.2$

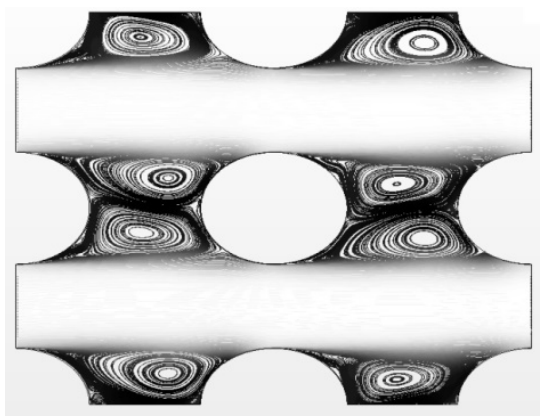

(d) $1.75 \times 1.75$

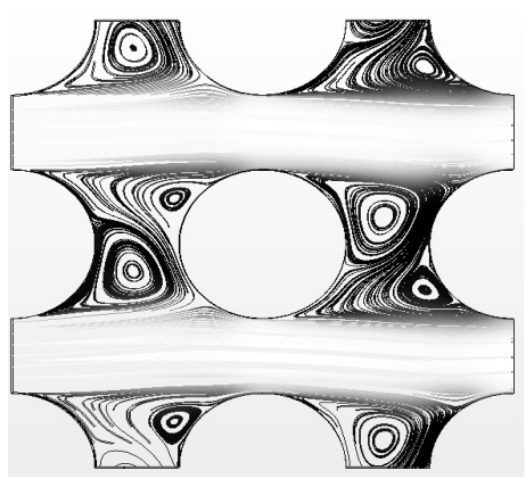

(b) $1.5 \times 1.5$

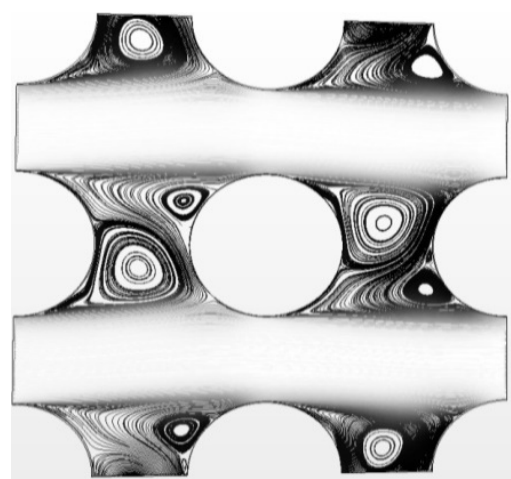

(c) $1.6 \times 1.6$

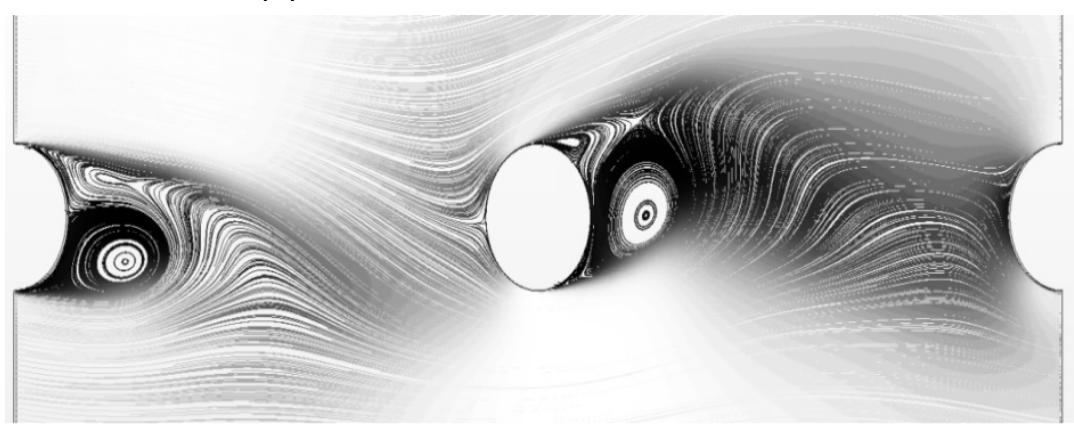

(e) $5 \times 5$

Figure 10: Mean velocity streamlines for the 2D square pitch ratios using the EB k- $\varepsilon$ model.

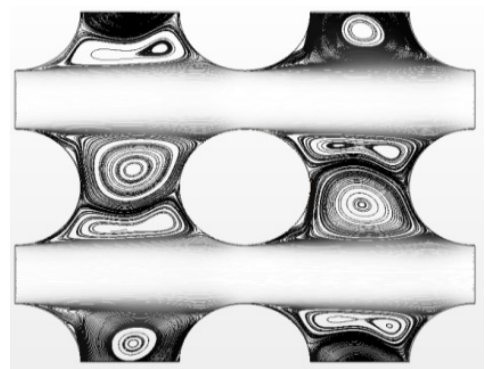

(a) $1.75 \times 1.5$

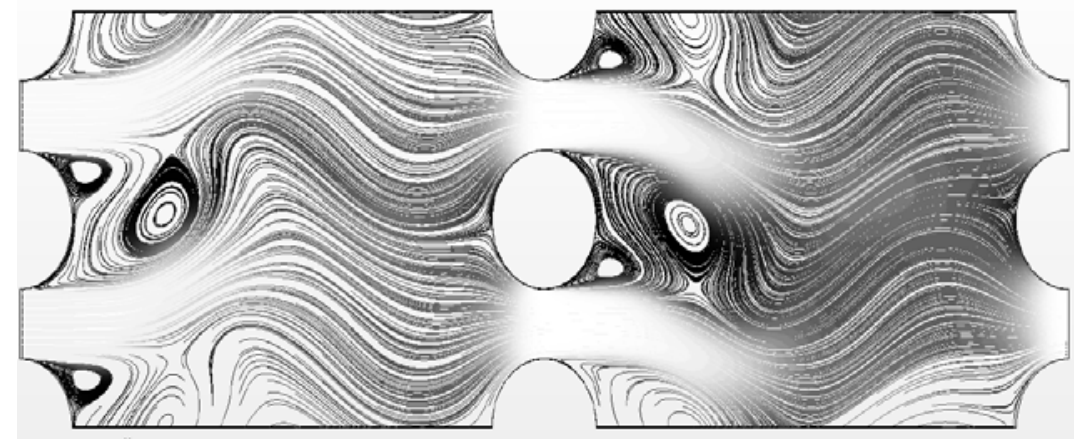

(c) $5 \times 1.5$

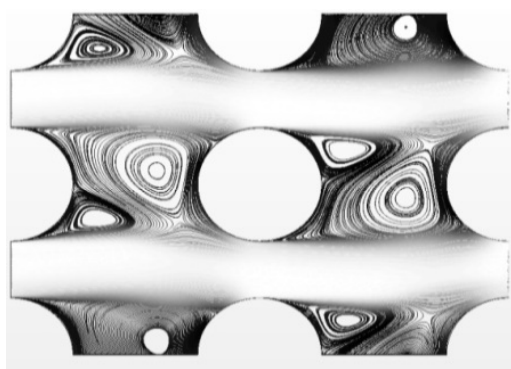

(b) $2 \times 1.5$ 


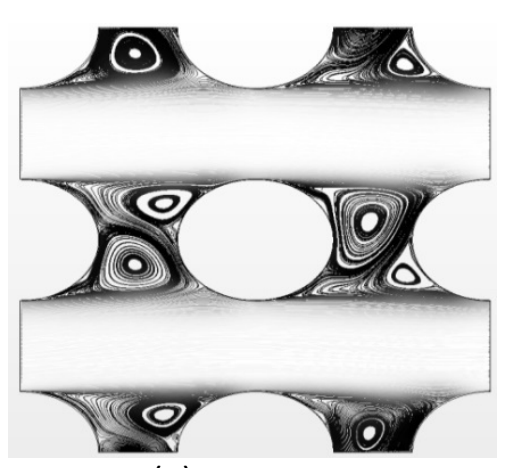

(a) $1.5 \times 1.75$

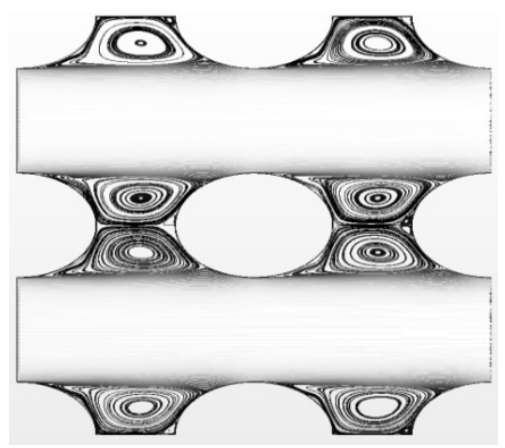

(b) $1.5 \times 2$

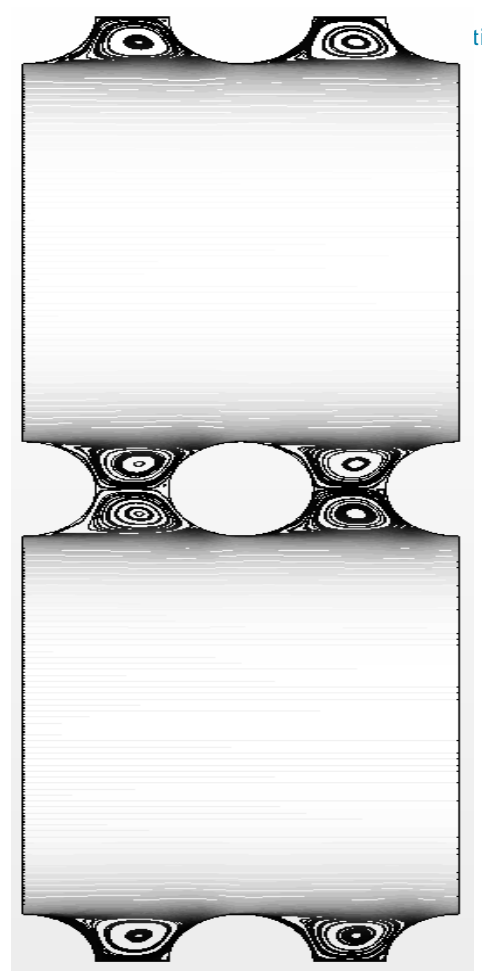

(c) $1.5 \times 5$

Figure 12: Mean velocity streamlines for non-square pitch ratios at a constant transvers distance using the EB k- $\varepsilon$ model.

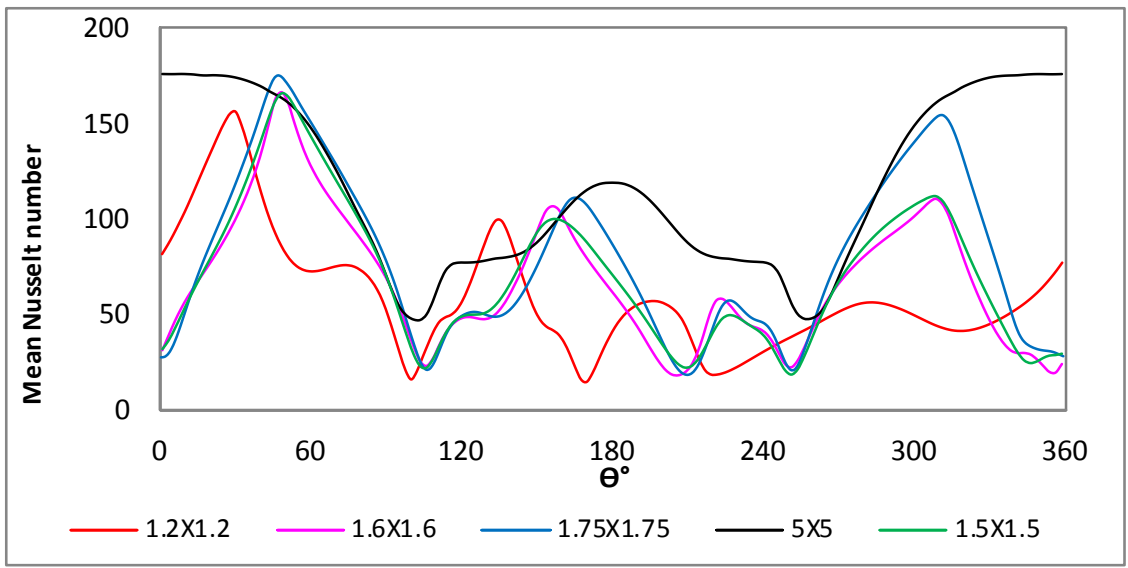

(a) 3D Square P.R.

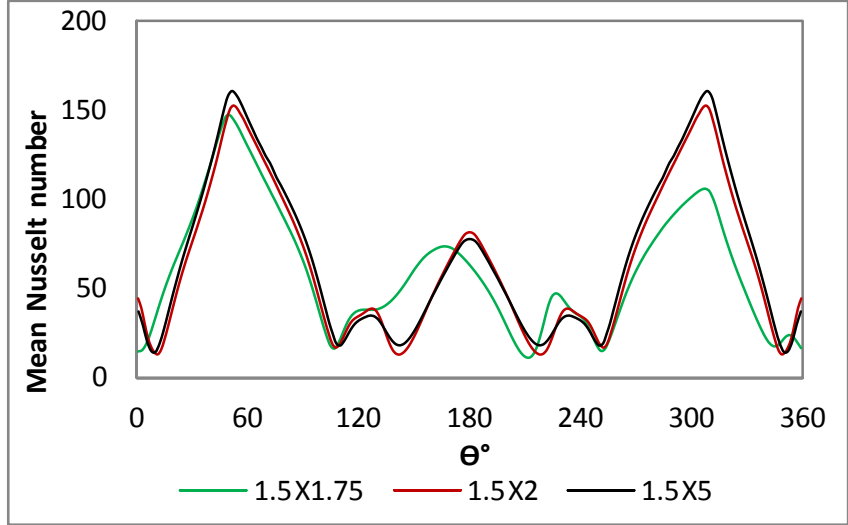

(b) 2D Non square P.R. at $X=$ constant

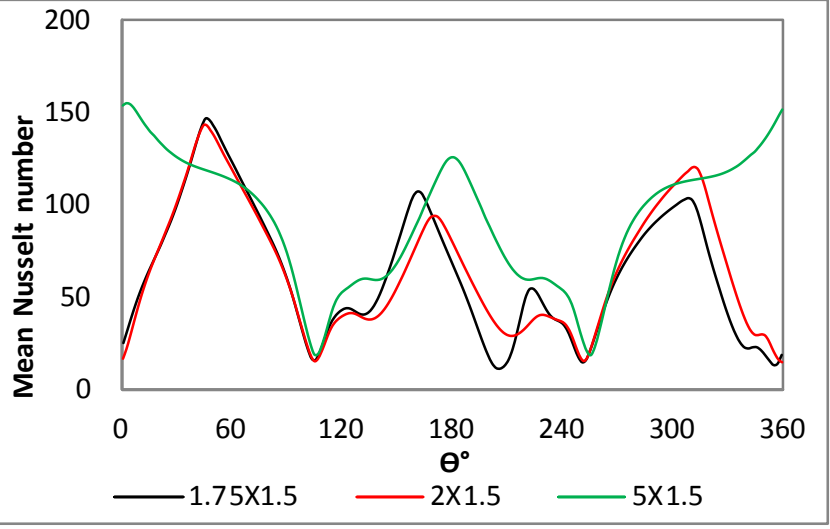

(c) 2D Non square P.R. at $Y=$ constant

Figure 13: Mean Nusselt number distributions around the central tube using the EB k- $\varepsilon$ model. 


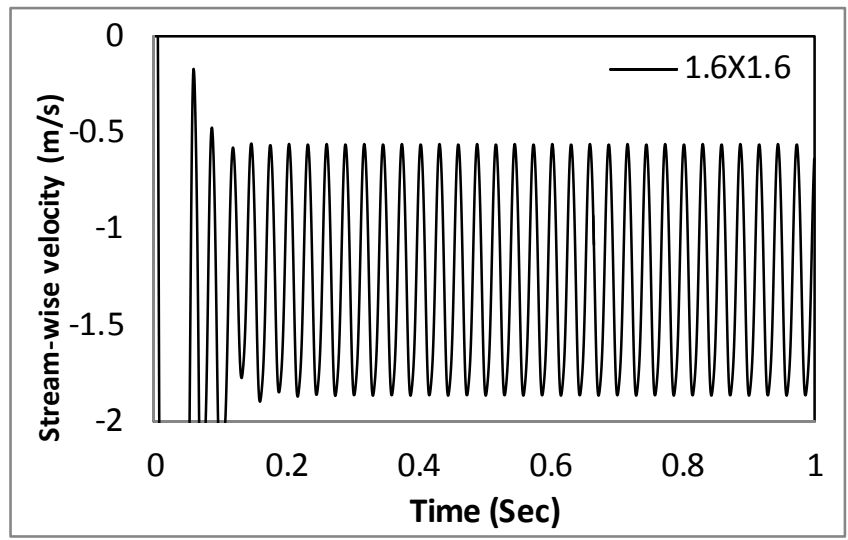

Figure 14: Vortex shedding frequency of the square pitch ratio of 1.6 in $3 \mathrm{D}$ using the $\mathrm{EB}$ k$\varepsilon$ model.

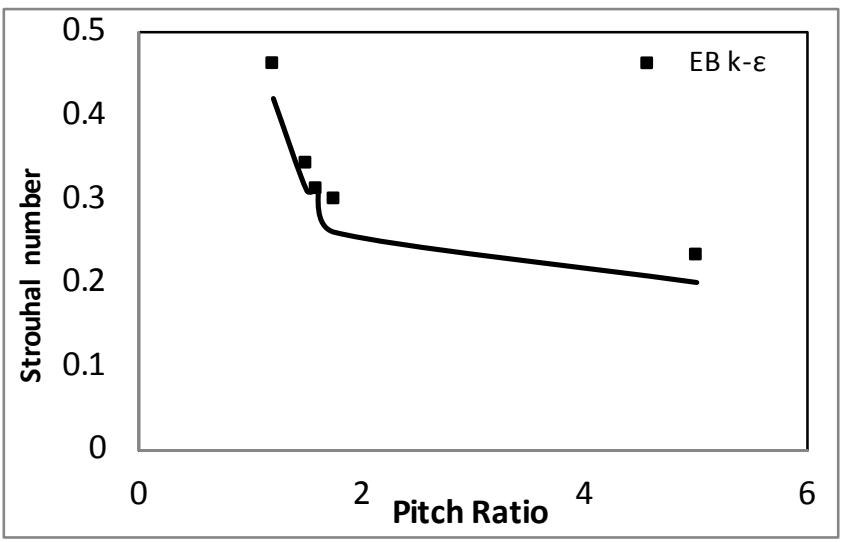

Figure 15: A comparison between experimental data of Fitz-Hugh (1937) and predicted results by the EB k- $\varepsilon$ model for 3D pitch square ratios.

\section{ACKNOWLEDGEMENTS}

I would like to thank the Higher Committee for the Development of Education in Iraq (HCDE) for supporting me financially and affording me the great opportunity to do the Master degree in the UK. However, my ID in this scholarship was (M1200705).

\section{Reference}

Aiba, S., Tsuchida, H., \& Ota, T. (1982). Heat Transfer Around Tubes in In-line Tube Banks. Bulletin of JSME. 25, 919-926.

Alastair West. "ASSESSMENT OF COMPUTATIONAL STRATEGIES FOR MODELLING IN-LINE TUBE BANKS". A thesis submitted to the University of Manchester for the degree of doctor of philosophy in The Faculty of Engineering and Physical Sciences, June 2013.

Beale, S. B. and D. B. Spalding (1999, August). A numerical study of unsteady fluid flow in in-line and staggered tube banks. Journal of Fluids and Structures 13(6), 723-754.

Benhamadouche, S., D. Laurence, N. Jarrin, I. Afgan, and C. Moulinec, 2005, "Large eddy simulation of flow across in -line tube bundles" In 11th International Topical Meeting on Nuclear Reactor Thermal Hydraulics .

Fitz-Hugh, J. S. (1973). Flow induced vibration in heat exchangers. Harwell, Berkshire, Atomic Energy Research Establishment.

Imran Afgan. "Large Eddy Simulation of cylindrical Bodies incorporating unstructured finite volume mesh". A thesis submitted to The University of Manchester for the degree of Doctor of Philosophy in the Faculty of Engineering and Physical Sciences, March 2007. 
Ishigai, S., Nishikawa, E., Yagi, E. 1973. Structures of Gas flow and vibration in tube banks with tube axes normal to flow. Inst. Sym. on Marine Engineering, Tokyo. PP. 1-5-23 to 1-5-33; in Flow induced vibration of circular cylinder structures by Chen, S. S.

Konstantinidis, E., Castiglia, D., Balabani, S., \& Yianneskis, M. (2000). On the Flow and Vortex Shedding Characteristics of an In-Line Tube Bundle in Steady and Pulsating Crossflow. Chemical Engineering Research and Design. 78, 1129-1138.

Ladjedel O., Adjlout L., Yahiaoui T., \& Imine O. (2013). CFD analysis of turbulent cross-flow in a staggered tube bundle equipped with grooved cylinders. EPJ Web of Conferences. 45.

Lam, K., Gong, W., \& So, R. (2008). Numerical simulation of cross-flow around four cylinders in an in-line square configuration. Journal of Fluids and Structures. 24, 34-57.

Lam, K., Lo, S. C. 1992. A visualization study of cross flow around four cylinders in a square configuration. J. Fluids Struct. 6. 109-131.

Liang, C. and Papadakis, G. (2005). Study of the Effect of Flow Pulsation on the Flow Field and Heat Transfer Over an Inline Cylinder Array Using LES.

Traub, D. (1990). Turbulent heat transfer and pressure drop in plain tube bundles. Chemical Engineering and Processing: Process Intensification. 28, 173-181.

Zdravkovich, M. M. 1987. THE EFFECTS OF INTERFERENCE BETWEEN CIRCULAR CYLINDERS IN CROSS FLOW. Journal of Fluids and Structures (1987) 1, 239-261. 